\title{
CONVERGENCE OF A TIME DISCRETIZATION FOR A CLASS OF NON-NEWTONIAN FLUID FLOW*
}

\author{
ETIENNE EMMRICH ${ }^{\dagger}$
}

Dedicated to Professor Rolf D. Grigorieff on the occasion of his 70th birthday

Abstract. The equation describing the non-stationary flow of an incompressible non-Newtonian fluid is approximated by the fully- and semi-implicit two-step backward differentiation formula (BDF). The stress tensor is assumed to be of $p$-structure such that the usual coercivity, growth, and monotonicity condition is fulfilled. Convergence of a piecewise polynomial prolongation of the discrete solution towards an exact weak solution is shown for the case $p \geq 1+2 d /(d+2)$, where $d$ denotes the spatial dimension.

Key words. Non-Newtonian fluid flow, weak solution, time discretization, two-step scheme, convergence, monotone operator.

AMS subject classifications. 76A05, 65M12, 47H05, 35Q35.

\section{Introduction}

Let $\Omega \subset \mathbb{R}^{d}(d \in\{2,3\})$ be an open bounded set with boundary $\partial \Omega$ of class $\mathcal{C}^{0,1}$, and let $(0, T)$ be the time interval under consideration. The flow of an incompressible fluid can be described by the initial-boundary value problem

$$
\begin{aligned}
& \partial_{t} u-\nabla \cdot \sigma+(u \cdot \nabla) u+\nabla p=f, \quad \nabla \cdot u=0 \quad \text { in } \Omega \times(0, T), \\
& u=0 \quad \text { on } \partial \Omega \times(0, T), \quad u(\cdot, 0)=u_{0} \quad \text { in } \Omega,
\end{aligned}
$$

where $u=u(x, t)$ denotes the velocity field with the prescribed initial velocity $u_{0}=$ $u_{0}(x), p=p(x, t)$ is the pressure, and $f=f(x, t)$ is an external force per unit mass. The symmetric stress tensor $\sigma=\sigma(e)$ is assumed to be a continuous function in the symmetrized velocity gradient $e(u)=\left(\nabla u+(\nabla u)^{T}\right) / 2$ and is assumed to fulfill the following structural assumption: There are numbers $p>1, \mu, c>0$ such that for all $y, z \in \mathbb{R}_{\mathrm{sym}}^{d \times d}$

$$
\sigma(z) \cdot z \geq \mu|z|^{p}, \quad|\sigma(z)| \leq c(1+|z|)^{p-1}, \quad(\sigma(z)-\sigma(y)) \cdot(z-y) \geq 0 .
$$

Note that $y \cdot z:=\sum_{i, j=1}^{d} y_{i j} z_{i j}$ for $y, z \in \mathbb{R}^{d \times d},|z|:=(z \cdot z)^{1 / 2}$, and $y \cdot z=y \cdot z^{T}$ if $y^{T}=y$. Typical examples are

- the Stokes law with $\sigma(z) \sim z$ that leads (with $p=q=2$ ) to the Navier-Stokes equation,

- power-law fluids with $\sigma(z) \sim|z|^{p-2} z$ that describe so-called shear thickening if $p>2$ and shear thinning if $1<p<2$, respectively, and

- variants of the power-law such as $\sigma(z) \sim\left(1+|z|^{2}\right)^{(p-2) / 2} z$.

A discussion of the non-Newtonian model can be found in standard monographs as e.g. $[4,7,27]$. Other than the model above, other descriptions of complex fluid flow that do not follow Newton's linear relation between stress and strain have been studied. Examples are viscoelastic fluid flow such as the Oldroyd model (see [14, 22,

\footnotetext{
${ }^{*}$ Received: April 7, 2008; accepted (in revised version): August 14, 2008. Communicated by Pingwen Zhang.

${ }^{\dagger}$ Technische Universität Berlin, Institut für Mathematik, Straße des 17. Juni 136, 10623 Berlin, Germany (emmrich@math.tu-berlin.de).
} 
28] and the references cited therein) and, more recently, the coupling of macroscopic and microscopic models for a multiscale description of polymeric liquids (see $[5,6$, $19,20,25]$ and the references cited therein). Some numerical analysis is available for these models but it is more focused on the spatial approximation.

Results on the (weak and measure-valued) solvability of the above model (1.1), (1.2) have been presented in detail in [23], see also the references cited therein. Recently, existence of weak solutions for $p>2 /(d+2)+2 d /(d+2)$ has been shown in [31] and for $p>2 d /(d+2)$ in [10]. In the case

$$
p \geq 1+\frac{2 d}{d+2}
$$

existence of a weak solution can be proven by employing Minty's trick for monotone operators (see also [21, pp. 207ff.]). This is, indeed, the method of proof, which we adapt here in order to prove convergence of a time discretization of (1.1), (1.2) under the restriction (1.3). The attempt of this paper is rather not to provide new results on existence but rather convergence of a numerical approximation in time.

Error estimates in the context of strong solutions (in the space-periodic case) have been obtained in $[9,26]$ for the fully- and semi-implicit Euler scheme combined with a finite element method. The analysis there relies upon the reasonable assumption that the stress tensor is the derivative of a potential. Moreover, the focus is on the interesting case $p \leq 2$. Semi-implicit methods may have some additional stabilizing impact (especially for small $p$ ), as is discussed in [9]. Indeed, only the diffusion term needs to be dealt with implicitly in order to cope with its stiffness (see $[17,30]$ for a discussion of stability requirements for dealing with stiff problems, see also [24, 29] in the context of the Navier-Stokes problem). Approximating the convection term explicitly may be advantageous, not only because the scheme then guarantees unique solvability but also due to lower computational costs. Implicit-explicit schemes have also been studied for other nonlinear evolution problems (see $[1,2,3]$ and the references cited therein).

In this paper, we consider the temporal semi-discretization of (1.1) by means of the widely used two-step BDF on an equidistant time grid. Let $\Delta t=T / N(N \in \mathbb{N})$ be the time step size. We then seek approximations $u^{n} \approx u\left(\cdot, t_{n}\right)\left(t_{n}=n \Delta t, n=2,3, \ldots, N\right)$ by replacing the time derivative by a divided difference,

$$
\partial_{t} u\left(\cdot, t_{n}\right) \approx \frac{1}{\Delta t}\left(\frac{3}{2} u^{n}-2 u^{n-1}+\frac{1}{2} u^{n-2}\right) .
$$

The diffusion term is dealt with implicitly whereas the convection term can also be dealt with explicitly. Note that the method is G-stable (see [17, p. 332]) and thus allows us to cope with stiff problems. Our proof, however, relies essentially on the algebraic relation from which the G-stability follows (see (3.5) below).

We prove convergence of piecewise polynomial prolongations of the discrete solution $\left\{u^{n}\right\}$ towards a weak solution to (1.1). It is obvious from the proof that the Euler scheme can be handled similarly, albeit somewhat easier. We shall not give the details here.

Standard references for time discretization methods are, e.g., [17, 30]. A detailed discussion of the literature on the analysis of the time discretization of nonlinear evolution equations of first order can be found in [13]. In [24, 29], the analysis of simple one-step methods applied to the Navier-Stokes problem is studied. A comparison of different numerical schemes for the approximation in time of the Navier-Stokes problem has recently been given in [18]. 
Recently, we were able to prove convergence of the two-step BDF for a rather large class of evolution equations governed by a monotone operator with a moderate, strongly continuous perturbation (see [13]). Unfortunately, the results cannot be applied directly to the problem under consideration here. This is due to the convection term. However, a modification of the method of proof allows us to study the problem considered here. Unlike [13], here we analyze the combination of the two-step BDF with an initial Euler step and also study a semi-implicit variant of this numerical scheme.

As in [13], we adapt Minty's monotonicity trick, and so we need that the time derivative is in the dual of the space in which the weak solution lies. This leads to the restriction (1.3) that $p$ has to be large enough. For the semi-implicit variant, we then also have uniqueness of the discrete solution.

Unfortunately, (1.3) does not include the case $p=2$ for $d=3$. However, in [11], we were able to prove convergence of the two-step BDF for the incompressible NavierStokes problem (with $p=2$ and $d \in\{2,3\}$ ). As the diffusion term then is linear, it is much easier to prove that convergence takes place in this term, and Minty's trick is not needed there. It remains open whether, e.g., the truncation technique used in [10, 31] can also be employed for proving convergence of a temporal semi-discretization of (1.1) if (1.3) is violated, especially in the interesting case $p \leq 2$.

Let us finally remark that we only consider the question of convergence and do not derive estimates for the error, as those estimates always rely upon the regularity of the exact solution (which is not known so far). This is different from the approach in $[9,26]$ for the Euler scheme applied to (1.1) and the approach in [12] for the twostep BDF applied to the Navier-Stokes problem relying upon its parabolic smoothing property. Convergence can already be shown in the framework of a weak solution, so we do not need to assume any further regularity. This is also the reason why we do not have to assume a potential structure for the stress tensor as in [26]. As the pressure then appears to be a distribution only, we do not consider its numerical approximation and only work in solenoidal function spaces.

Nevertheless, it is of interest to consider an efficient higher-order method (the twostep BDF is of second order when applied to, e.g., a linear parabolic problem with sufficiently smooth solution, and its computational costs are not much higher than for the Euler scheme) as the exact solution to (1.1) may exhibit some (local) regularity depending on the smoothness and compatibility of the problem data. It would be favorable to have results for a variable step size/variable order BDF implementation (see $[15,16])$ that can adaptively deal with local regularity. This is, however, out of scope so far. In this regard, the results of the paper in hand may be seen as preliminary results.

For a full discretization, we need to combine the two-step BDF with an appropriate spatial approximation scheme. As long as the spatial approximation is a conforming method, it is expected that the techniques employed here carry over. Non-conforming methods surely require a detailed study of their own. See [26] for a detailed error analysis in the spatially periodic case of both conforming and nonconforming finite element methods. See [29] for convergence results of internal and external approximation schemes for the Navier-Stokes problem.

The paper is organized as follows. In section 2 we present the notation and auxiliary results concerning the functional analytic framework. In section 3 , we introduce the numerical scheme and study its properties. The main part is section 4 , in which we prove the convergence of the method. 


\section{Notation and auxiliary results}

Throughout this paper, we make use of the standard notation for Lebesgue, Sobolev, Bochner-Lebesgue spaces and spaces of continuous functions.

Let $V$ and $H$ be the closure of $\mathcal{V}:=\left\{v \in \mathcal{C}_{0}^{\infty}(\Omega)^{d}: \nabla \cdot v=0\right\}$ with respect to the $W^{1, p}$-norm and $L^{2}$-norm, respectively. If $\partial \Omega$ is of class $\mathcal{C}^{0,1}$, we have

$$
V=\left\{v \in W_{0}^{1, p}(\Omega)^{d}: \nabla \cdot v=0\right\}, \quad H=\left\{v \in L^{2}(\Omega)^{d}: \nabla \cdot v=0, \gamma_{n} v=0\right\},
$$

where $\gamma_{n}$ denotes the trace in the normal direction (see [21, p. 209], [29, pp. 13ff.]). The norm in $V$ shall be denoted by $\|\cdot\|$, whereas $|\cdot|$ denotes the norm in $H$. We always assume $p>2 d /(d+2)$ as then $V, H$, and the dual space $V^{*}$ (with $\|\cdot\|_{*}$ and $\langle\cdot, \cdot\rangle$ denoting the dual norm and pairing, respectively) form a Gelfand triple with compact embeddings. We denote the exponent conjugated to $p$ by $p^{*}:=p /(p-1)$. Note that

$$
L^{p}(0, T ; V) \subset L^{2}(0, T ; H) \subset L^{p^{*}}\left(0, T ; V^{*}\right)
$$

also forms a Gelfand triple if $p \geq 2$.

For Lebesgue exponents $p, q \in[1, \infty]$, we set

$$
\mathcal{W}^{p, q}(0, T):=\left\{v \in L^{p}(0, T ; V): v^{\prime} \in L^{q}\left(0, T ; V^{*}\right)\right\},
$$

which is a Banach space when equipped with the norm $\|v\|_{\mathcal{W}^{p, q}(0, T)}:=\|v\|_{L^{p}(0, T ; V)}+$ $\left\|v^{\prime}\right\|_{L^{q}\left(0, T ; V^{*}\right)}$. By $v^{\prime}$, we denote the distributional time derivative. Note that

$$
\mathcal{W}^{1,1}(0, T) \hookrightarrow \mathcal{C}\left([0, T] ; V^{*}\right), \quad \mathcal{W}^{p, p^{*}}(0, T) \hookrightarrow \mathcal{C}([0, T] ; H) .
$$

As $V \stackrel{c}{\hookrightarrow} H$, we also know by the compactness theorem of Lions-Aubin (see [21, Thm. 5.1 on p. 58]) that

$$
\mathcal{W}^{p, q}(0, T) \stackrel{c}{\hookrightarrow} L^{p}(0, T ; H), \quad p>1, q \geq 1 .
$$

The following interpolatory inequalities are rather well known and can be proved by employing Hölder's inequality and Sobolev's embedding theorem (see [8, pp. 194f.]).

Lemma 2.1. Let $r \geq 2$. If $p \geq d$ then, for any $\theta \in\left[2\left(\frac{1}{2}-\frac{1}{r}\right), 1\right]$, there is a constant $c>0$ such that for all $v \in V$

$$
\|v\|_{0, r} \leq c|v|^{1-\theta}\|v\|^{\theta} .
$$

If $\frac{r d}{d+r} \leq p<d$ then there is a constant $c>0$ such that for all $v \in V$

$$
\|v\|_{0, r} \leq c|v|^{1-\theta}\|v\|^{\theta} \text { with } \theta=\left(\frac{1}{2}+\frac{1}{d}-\frac{1}{p}\right)^{-1}\left(\frac{1}{2}-\frac{1}{r}\right) .
$$

Here and in what follows, $c$ denotes a generic positive constant. Also well known is Korn's first inequality (see [23, Thm. 1.10 on p. 196]).

LEMmA 2.2. There is a constant $c>0$ such that for all $v \in W_{0}^{1, p}(\Omega)^{d}(p \in(1, \infty))$

$$
\|v\|_{1, p} \leq c\|e(v)\|_{0, p}
$$


With the diffusion term, we associate the nonlinear operator

$$
A: V \rightarrow V^{*}, \quad\langle A v, w\rangle=\int_{\Omega} \sigma(e(v)) \cdot \nabla w d x=\int_{\Omega} \sigma(e(v)) \cdot e(w) d x, \quad v, w \in V .
$$

LEMMA 2.3. For the symmetric, continuous stress tensor function $\sigma$, assume (1.2). Then $A: V \rightarrow V^{*}$ is monotone, hemicontinuous, coercive, and fulfills a growth condition. There are constants $\mu, c>0$ such that for all $v \in V$

$$
\langle A v, v\rangle \geq \mu\|v\|^{p}, \quad\|A v\|_{*} \leq c(1+\|v\|)^{p-1} .
$$

$\operatorname{Via}(A u)(t):=A u(t)$ for $u:[0, T] \rightarrow V$, the operator $A: V \rightarrow V^{*}$ extends to an operator

$$
A: L^{p}(0, T ; V) \rightarrow L^{p^{*}}\left(0, T ; V^{*}\right)
$$

that is monotone, hemicontinuous, coercive, and fulfills a growth condition such that for all $v \in L^{p}(0, T ; V)$

$$
\langle A v, v\rangle \geq \mu\|v\|_{L^{p}(0, T ; V)}^{p}, \quad\|A v\|_{L^{p^{*}}\left(0, T ; V^{*}\right)} \leq c\left(1+\|v\|_{L^{p}(0, T ; V)}\right)^{p-1} .
$$

Proof. The first part immediately follows from the assumptions on $\sigma$ and Korn's first inequality (see Lemma 2.2). For the second part, we observe that $A: V \rightarrow V^{*}$ is demicontinuous and thus maps Bochner measurable functions $u:[0, T] \rightarrow V$ into Bochner measurable functions $A u:[0, T] \rightarrow V^{*}$. The integrability then follows from the growth condition. The rest of the proof follows standard arguments (see [32, Lemma 30.2]).

For more details in the case of $A$ being a potential operator, see also [23, pp. 193ff.].

For sufficiently smooth functions $u, v, w$, let

$$
b(u, v, w):=\int_{\Omega}(u \cdot \nabla) v \cdot w d x .
$$

The trilinear form $b(\cdot, \cdot, \cdot)$ fulfills the usual estimates as known from [21, 29]. We may then define the operators

$$
\begin{aligned}
& B(\cdot, \cdot): V \times V \rightarrow V^{*}, \quad\langle B(u, v), w\rangle=b(u, v, w), \quad u, v, w \in V, \\
& B: V \rightarrow V^{*}, \quad B v=B(v, v),
\end{aligned}
$$

that describe the convection term. Note that $b(\cdot, \cdot, \cdot)$ is skew-symmetric in the last two arguments if the first argument is in $V$. We thus have in particular

$$
\langle B(u, v), v\rangle=\langle B v, v\rangle=0 \quad \forall u, v \in V .
$$

LEMmA 2.4. Let $p \geq \frac{3 d}{d+2}$ and

$$
\frac{1}{p} \leq \theta \leq 1 \text { if } p \geq d, \quad \theta=\left(\frac{1}{2}+\frac{1}{d}-\frac{1}{p}\right)^{-1} \frac{1}{2 p} \text { if } p<d
$$


Then there is a constant $c>0$ such that for all $u, v \in V$

$$
\|B v\|_{*} \leq c|v|^{2(1-\theta)}\|v\|^{2 \theta}
$$

as well as

$$
\|B u-B v\|_{*} \leq c|u-v|^{1-\theta}\|u-v\|^{\theta}\left(|u|^{1-\theta}\|u\|^{\theta}+|v|^{1-\theta}\|v\|^{\theta}\right) .
$$

The operator $B: V \rightarrow V^{*}$ is strongly continuous.

Let, in addition,

$$
p \geq \frac{1+\sqrt{5}}{2} \text { if } d=2, \quad p \geq \frac{3+\sqrt{39}}{5} \text { if } d=3 .
$$

$\operatorname{Via}(B u)(t):=B u(t)$ for $u:[0, T] \rightarrow V$, the operator $B: V \rightarrow V^{*}$ then extends to an operator

$$
\begin{aligned}
& B: L^{\infty}(0, T ; H) \cap L^{p}(0, T ; V) \rightarrow L^{r}\left(0, T ; V^{*}\right) \\
& \text { with } r=\frac{p^{2}}{2} \geq 1 \text { if } p \geq d, \quad r=\left(\frac{1}{2}+\frac{1}{d}-\frac{1}{p}\right) p^{2} \geq 1 \text { if } p<d .
\end{aligned}
$$

Moreover, there is a constant $c>0$ such that for all $v \in L^{\infty}(0, T ; H) \cap L^{p}(0, T ; V)$

$$
\|B v\|_{L^{r}\left(0, T ; V^{*}\right)} \leq c|v|_{L^{\infty}(0, T ; H)}^{2-\frac{p}{r}}\|v\|_{L^{p}(0, T ; V)}^{\frac{p}{r}} .
$$

If (1.3) holds then $r \geq p^{*}$. Finally, for any $q \in\left(1, \frac{p}{2 \theta}\right)$ (with $\theta$ as above), there is a constant $c>0$ such that for all $u, v \in L^{\infty}(0, T ; H) \cap L^{p}(0, T ; V)$

$$
\begin{aligned}
& \|B u-B v\|_{L^{q}\left(0, T ; V^{*}\right)} \leq c\|u-v\|_{L^{\bar{q}}(0, T ; H)}^{1-\theta}\|u-v\|_{L^{p}(0, T ; V)}^{\theta} \times \\
& \left(\|u\|_{L^{\infty}(0, T ; H)}^{1-\theta}\|u\|_{L^{p}(0, T ; V)}^{\theta}+\|v\|_{L^{\infty}(0, T ; H)}^{1-\theta}\|v\|_{L^{p}(0, T ; V)}^{\theta}\right), \quad \text { with } \bar{q}=\frac{(1-\theta) p q}{p-2 \theta q}>1 .
\end{aligned}
$$

Proof. With $b(v, v, w)=-b(v, w, v)$ for $v, w \in V$, Hölder's inequality, and Lemma 2.1 with $r=2 p /(p-1)$, we immediately find the growth estimate $(2.3)$. With

$$
\langle B u-B v, w\rangle=-b(u-v, w, u)-b(v, w, u-v)
$$

Hölder's inequality, and Lemma 2.1 with $r=2 p /(p-1)$, we obtain (2.4). The strong continuity of $B: V \rightarrow V^{*}$ follows from (2.4) since $V$ is compactly embedded in $H$.

Let $u=u(t) \in L^{\infty}(0, T ; H) \cap L^{p}(0, T ; V)$. Because of the foregoing estimates, $B u$ : $[0, T] \rightarrow V^{*}$ is Bochner measurable. The integrability of $t \mapsto\|B u(t)\|_{*}^{r}$ follows from the growth estimate (2.3) if $2 \theta r \leq p$. Here, in order to have $r \geq 1$, we need to assume (2.5). This also yields (2.6). The estimate (2.7) follows from (2.4) and Hölder's inequality.

\section{Temporal semi-discretization}

Assuming (1.3), we can now consider the weak formulation of (1.1) that, in the usual way (see $[21,29,32]$ for more details), leads to the evolution problem

$$
u^{\prime}+A u+B u=f \text { in } L^{p^{*}}\left(0, T ; V^{*}\right), \quad u(0)=u_{0} \in H .
$$


The main idea behind this is to have a solution $u \in L^{\infty}(0, T ; H) \cap L^{p}(0, T ; V)$ (the existence of which can be proven by means of a Galerkin method or even by a temporal semi-discretization as done in this paper) and then to show, by employing the growth estimates for $A$ and $B$ (see, in particular, estimate (2.6)), that the distributional time derivative $u^{\prime}$ of $u$ is indeed an element of $L^{p^{*}}\left(0, T ; V^{*}\right) \cong\left(L^{p}(0, T ; V)\right)^{*}$. The initial condition then makes sense because of the continuous embedding $\mathcal{W}^{p, p^{*}}(0, T) \hookrightarrow$ $\mathcal{C}([0, T] ; H)$.

Problem (3.1) shall now be discretized in time: For $N \in \mathbb{N}$, let $\Delta t=T / N, t_{n}=n \Delta t$ $(n=0,1, \ldots, N)$. Since the two-step BDF requires two starting values $u^{0}, u^{1}$, we employ one Euler step in order to compute $u^{1}$ from $u^{0}$. For simplicity, we only consider the natural restriction of $f$ as the approximation of the right-hand side. So, for given $u_{0} \in H$ and $f \in L^{p^{*}}\left(0, T ; V^{*}\right)$, we consider the numerical scheme

$$
\begin{aligned}
& \left.u^{0} \in H \text { given (with } u^{0} \approx u_{0}\right), \\
& \frac{1}{\Delta t}\left(u^{1}-u^{0}\right)+A u^{1}+B u^{1}=f^{1}:=\frac{1}{\Delta t} \int_{0}^{t_{1}} f(t) d t, \\
& \frac{1}{\Delta t}\left(\frac{3}{2} u^{n}-2 u^{n-1}+\frac{1}{2} u^{n-2}\right)+A u^{n}+B u^{n}=f^{n} \\
& \text { with } f^{n}:=\frac{3}{2 \Delta t} \int_{t_{n-1}}^{t_{n}} f(t) d t-\frac{1}{2 \Delta t} \int_{t_{n-2}}^{t_{n-1}} f(t) d t, \quad n=2,3, \ldots, N .
\end{aligned}
$$

We also consider a semi-implicit variant of the scheme (3.2) in which we replace $B u^{1}=B\left(u^{1}, u^{1}\right)$ by $B\left(u^{0}, u^{1}\right)$ and $B u^{n}=B\left(u^{n}, u^{n}\right)$ by $B\left(2 u^{n-1}-u^{n-2}, u^{n}\right)$ $(n=2,3, \ldots, N)$. Note that $2 u\left(t_{n-1}\right)-u\left(t_{n-2}\right)$ is, for smooth $u$, a second-order extrapolation to $u\left(t_{n}\right)$. This gives rise to the use of $2 u^{n-1}-u^{n-2}$ in the approximation. For short, we denote

$$
B u^{n} \leadsto B\left(E u^{n}, u^{n}\right) \text { with } E u^{1}=u^{0}, E u^{n}=2 u^{n-1}-u^{n-2}(n=2,3, \ldots, N) .
$$

For the modified scheme, we have to require $u^{0} \in V$ (which is always possible for $u_{0} \in H$ as $V$ is dense in $H$, see also the convergence result in the next section).

THEOREM 3.1. Assume (1.2) and (1.3). Then there is a solution $\left\{u^{n}\right\}_{n=1}^{N} \subset V$ to (3.2) that satisfies the following a priori estimates for $n=1,2, \ldots, N$ :

$$
\begin{aligned}
& \left|u^{n}\right|^{2}+\left|u^{1}-u^{0}\right|^{2}+\sum_{j=2}^{n}\left|u^{j}-2 u^{j-1}+u^{j-2}\right|^{2}+\mu \Delta t \sum_{j=1}^{n}\left\|u^{j}\right\|^{p} \\
& \leq c\left(\left|u^{0}\right|^{2}+\|f\|_{L^{p^{*}\left(0, T ; V^{*}\right)}}^{p^{*}}\right)=: M\left(u^{0}, f\right), \\
& \Delta t\left\|\frac{1}{\Delta t}\left(u^{1}-u^{0}\right)\right\|_{*}^{p^{*}}+\Delta t \sum_{j=2}^{n}\left\|\frac{1}{\Delta t}\left(\frac{3}{2} u^{j}-2 u^{j-1}+\frac{1}{2} u^{j-2}\right)\right\|_{*}^{p^{*}} \leq M^{\prime}\left(u^{0}, f\right),
\end{aligned}
$$

where $M^{\prime}\left(u^{0}, f\right)$ is a function in $M\left(u^{0}, f\right)$ that is bounded on bounded subsets. The assertions also apply to the semi-implicit variant of (3.2) with (3.3), where $M^{\prime}\left(u^{0}, f\right)$ now is a function in $M\left(u^{0}, f\right)+\Delta t\left\|u^{0}\right\|^{p}$ that is bounded on bounded subsets. The semi-implicit scheme is, in addition, uniquely solvable.

Proof. Existence of a solution to (3.2) follows step-by-step from Brézis' theorem on pseudomonotone operators (see [32, Thm. 27.A]) since $A: V \rightarrow V^{*}$ is monotone and 
hemicontinuous, $B: V \rightarrow V^{*}$ is strongly continuous, and the sum $A+B$ is coercive, see also (2.1), Lemma 2.3, 2.4.

In order to show the first a priori estimate, we test the equations with $u^{n}$, employ the identities

$$
\begin{aligned}
& (a-b) a=\frac{1}{2}\left(a^{2}-b^{2}+(a-b)^{2}\right), \\
& \left(\frac{3}{2} a-2 b+\frac{1}{2} c\right) a=\frac{1}{4}\left(a^{2}+(2 a-b)^{2}-b^{2}-(2 b-c)^{2}+(a-2 b+c)^{2}\right), \quad a, b, c \in \mathbb{R},
\end{aligned}
$$

the coercivity of $A$ as well as (2.1), and apply Young's inequality. This yields

$$
\frac{1}{2}\left|u^{1}\right|^{2}-\frac{1}{2}\left|u^{0}\right|^{2}+\frac{1}{2}\left|u^{1}-u^{0}\right|^{2}+\frac{\mu \Delta t}{2}\left\|u^{1}\right\|^{p} \leq c \Delta t\left\|f^{1}\right\|_{*}^{p^{*}}
$$

and, for $n=2,3, \ldots, N$,

$$
\begin{aligned}
& \frac{1}{4}\left|u^{n}\right|^{2}+\frac{1}{4}\left|2 u^{n}-u^{n-1}\right|^{2}-\frac{1}{4}\left|u^{n-1}\right|^{2}-\frac{1}{4}\left|2 u^{n-1}-u^{n-2}\right|^{2} \\
& +\frac{1}{4}\left|u^{n}-2 u^{n-1}+u^{n-2}\right|^{2}+\frac{\mu \Delta t}{4}\left\|u^{n}\right\|^{p} \leq c \Delta t\left\|f^{n}\right\|_{*}^{p^{*}} .
\end{aligned}
$$

We then sum up (taking twice the equation for the initial Euler step), where we observe for the remaining initial terms that

$$
\left|u^{1}\right|^{2}-\left|u^{0}\right|^{2}+\left|u^{1}-u^{0}\right|^{2}-\frac{1}{4}\left(\left|u^{1}\right|^{2}+\left|2 u^{1}-u^{0}\right|^{2}\right)=\frac{1}{4}\left|u^{1}\right|^{2}+\frac{1}{2}\left|u^{1}-u^{0}\right|^{2}-\frac{3}{4}\left|u^{0}\right|^{2} .
$$

This leads, after multiplication by 4 , to

$$
\begin{gathered}
\left|u^{n}\right|^{2}+2\left|u^{1}-u^{0}\right|^{2}+\sum_{j=2}^{n}\left|u^{j}-2 u^{j-1}+u^{j-2}\right|^{2}+\mu \Delta t \sum_{j=1}^{n}\left\|u^{j}\right\|^{p} \\
\leq c\left(\left|u^{0}\right|^{2}+\Delta t \sum_{j=1}^{n}\left\|f^{j}\right\|_{*}^{p^{*}}\right)
\end{gathered}
$$

With Hölder's inequality, we also find

$$
\Delta t \sum_{j=1}^{n}\left\|f^{j}\right\|_{*}^{p^{*}} \leq c \int_{0}^{t_{n}}\|f(t)\|_{*}^{p^{*}} d t
$$

which, finally, proves the first estimate.

The second estimate follows from the growth estimates for $A$ and $B$ together with the first estimate. From the numerical scheme (3.2), we obtain for $n=1,2, \ldots, N$ with 
Lemma 2.3 and $(2.3)$

$$
\begin{aligned}
& \Delta t\left\|\frac{1}{\Delta t}\left(u^{1}-u^{0}\right)\right\|_{*}^{p^{*}}+\Delta t \sum_{j=2}^{n}\left\|\frac{1}{\Delta t}\left(\frac{3}{2} u^{j}-2 u^{j-1}+\frac{1}{2} u^{j-2}\right)\right\|_{*}^{p^{*}} \\
& =\Delta t\left\|f^{1}-A u^{1}-B u^{1}\right\|_{*}^{p^{*}}+\Delta t \sum_{j=2}^{n}\left\|f^{j}-A u^{j}-B u^{j}\right\|_{*}^{p^{*}} \\
& \leq c \Delta t \sum_{j=1}^{n}\left\|f^{j}\right\|_{*}^{p^{*}}+c \Delta t \sum_{j=1}^{n}\left\|A u^{j}\right\|_{*}^{p^{*}}+c \Delta t \sum_{j=1}^{n}\left\|B u^{j}\right\|_{*}^{p^{*}} \\
& \leq c \Delta t \sum_{j=1}^{n}\left\|f^{j}\right\|_{*}^{p^{*}}+c \Delta t \sum_{j=1}^{n}\left(1+\left\|u^{j}\right\|\right)^{p}+c \Delta t \sum_{j=1}^{n}\left|u^{j}\right|^{2(1-\theta) p^{*}}\left\|u^{j}\right\|^{2 \theta p^{*}}
\end{aligned}
$$

with $\theta$ given by (2.2). Since $2 \theta p^{*} \leq p$ for (1.3) (if $d=2$ then $\theta$ can be chosen in such a way), Hölder's inequality together with the first a priori estimate proves the assertion.

For each time step of the semi-implicit variant, we can apply the Browder-Minty theorem (see [32, Thm. 26.A]). Since, in particular,

$$
\left\langle B\left(E u^{n}, v\right)-B\left(E u^{n}, w\right), v-w\right\rangle=b\left(E u^{n}, v-w, v-w\right)=0, \quad v, w \in V, n=1,2, \ldots, N
$$

the operator appearing in each step turns out to be strictly monotone, and thus, the solution is uniquely determined. The a priori estimates follow as before. For the second one, note that

$$
\left\|B\left(E u^{j}, u^{j}\right)\right\|_{*} \leq c\left|E u^{j}\right|^{1-\theta}\left\|E u^{j}\right\|^{\theta}\left|u^{j}\right|^{1-\theta}\left\|u^{j}\right\|^{\theta}
$$

and thus

$$
\begin{aligned}
& \Delta t \sum_{j=1}^{n}\left\|B\left(E u^{j}, u^{j}\right)\right\|_{*}^{p^{*}} \\
& \leq c \max _{j=1,2, \ldots, n}\left|E u^{j}\right|^{(1-\theta) p^{*}}\left|u^{j}\right|^{(1-\theta) p^{*}}\left(\Delta t \sum_{j=1}^{n}\left\|u^{j}\right\|^{p}\right)^{\theta p^{*} / p}\left(\Delta t \sum_{j=1}^{n}\left\|E u^{j}\right\|^{p}\right)^{\theta p^{*} / p} \\
& \leq c \max _{j=0,1, \ldots, n}\left|u^{j}\right|^{2(1-\theta) p^{*}}\left(\Delta t \sum_{j=0}^{n}\left\|u^{j}\right\|^{p}\right)^{2 \theta p^{*} / p} \cdot
\end{aligned}
$$

\section{Convergence}

From the discrete solution $\left\{u^{n}\right\}$ on the time grid with the time step $\Delta t$, we now construct piecewise polynomial functions that are defined on the whole interval $[0, T]$ :

$$
\begin{aligned}
& u_{\Delta t}(0)= u^{1}, \quad u_{\Delta t}(t)=u^{n} \text { if } t \in\left(t_{n-1}, t_{n}\right](n=1,2, \ldots, N), \\
& v_{\Delta t}(t)=\left\{\begin{array}{l}
\frac{3}{2} u^{1}-\frac{1}{2} u^{0}+\left(u^{1}-u^{0}\right) \frac{t-t_{1}}{\Delta t} \text { if } t \in\left[0, t_{1}\right] \\
\frac{3}{2} u^{n}-\frac{1}{2} u^{n-1}+\left(\frac{3}{2} u^{n}-2 u^{n-1}+\frac{1}{2} u^{n-2}\right) \frac{t-t_{n}}{\Delta t} \\
\text { if } t \in\left(t_{n-1}, t_{n}\right](n=2,3, \ldots, N) .
\end{array}\right.
\end{aligned}
$$

This construction reflects the choice of the method as the slope of $v_{\Delta t}$ is just the divided difference that is used to replace the time derivative in order to obtain the 
numerical scheme. Note, however, that the continuous function $v_{\Delta t}$ does not interpolate.

In the following, all quantities that are related to a time grid with the time step $\Delta t=T / N$ are denoted by a subscript $\Delta t$ (except $N$ and the grid points $\left.t_{n}\right)$.

The main result of this paper now reads as follows.

TheOREM 4.1. Assume (1.2) and (1.3). Let $\left\{(\Delta t)_{k}\right\}$ be a null sequence of time steps $(\Delta t)_{k}=T / N_{k}$ with $\left\{N_{k}\right\} \subset \mathbb{N}, N_{k} \rightarrow \infty$, and let $\left\{u_{(\Delta t)_{k}}\right\},\left\{v_{(\Delta t)_{k}}\right\}$ be the corresponding sequences of the polynomial prolongations (4.1) of the discrete solutions to the scheme (3.2) or its semi-implicit variant with

$$
\left\{u_{(\Delta t)_{k}}^{0}\right\} \subset V, \quad u_{(\Delta t)_{k}}^{0} \rightarrow u_{0} \text { in } H, \quad(\Delta t)_{k}\left\|u_{(\Delta t)_{k}}^{0}\right\|^{p} \leq c
$$

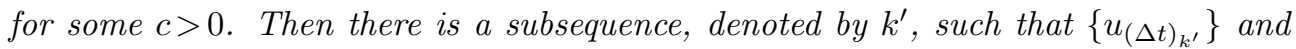
$\left\{v_{(\Delta t)_{k^{\prime}}}\right\}$ converge weakly in $L^{p}(0, T ; V)$, weakly* in $L^{\infty}(0, T ; H)$ as well as strongly in $L^{r}(0, T ; H)$ for any $r \in[1, \infty)$ towards a solution $u \in \mathcal{W}^{p, p^{*}}(0, T)$ to (3.1). The subsequence of time derivatives $v_{(\Delta t)_{k^{\prime}}}$ also converges weakly in $L^{p^{*}}\left(0, T ; V^{*}\right)$ towards the derivative $u^{\prime}$.

The assumption (4.2) on the corresponding sequence of initial data can always be fulfilled since $V$ is dense in $H \ni u_{0}$. The proof of Theorem 4.1 will be prepared by the following lemmas. An immediate consequence of the a priori estimates in Theorem 3.1 is

LEMma 4.2. Under the assumptions of Theorem 4.1, there is a function $u \in$ $\mathcal{W}^{p, p^{*}}(0, T)$ and a subsequence, denoted by $k^{\prime}$, such that

$$
\begin{aligned}
& u_{(\Delta t)_{k^{\prime}}} \rightarrow u \text { in } L^{p}(0, T ; V), u_{(\Delta t)_{k^{\prime}}} \stackrel{*}{\rightarrow} u \text { in } L^{\infty}(0, T ; H), \\
& v_{(\Delta t)_{k^{\prime}}} \rightarrow u \text { in } L^{p}(0, T ; V), v_{(\Delta t)_{k^{\prime}}} \stackrel{*}{\rightarrow} u \text { in } L^{\infty}(0, T ; H), \\
& v_{(\Delta t)_{k^{\prime}}}^{\prime} \rightarrow u^{\prime} \text { in } L^{p^{*}}\left(0, T ; V^{*}\right), v_{(\Delta t)_{k^{\prime}}} \rightarrow u \text { in } L^{r}(0, T ; H) \forall r \in[1, \infty) .
\end{aligned}
$$

Proof. For readability, we omit the subscript $k$. It is straightforward to prove, with Theorem 3.1, the boundedness of $\left\{u_{\Delta t}\right\}$ and $\left\{v_{\Delta t}\right\}$ in $L^{p}(0, T ; V) \cap L^{\infty}(0, T ; H)$ and of $\left\{v_{\Delta t}^{\prime}\right\}$ in $L^{p^{*}}\left(0, T ; V^{*}\right)$. In particular, we find

$$
\begin{aligned}
& \left\|v_{\Delta t}\right\|_{L^{p}(0, T ; V)}^{p}=\sum_{n=1}^{N} \int_{t_{n-1}}^{t_{n}}\left\|v_{\Delta t}(t)\right\|^{p} d t=\int_{0}^{\Delta t}\left\|\frac{3}{2} u^{1}-\frac{1}{2} u^{0}+\left(u^{1}-u^{0}\right) \frac{t-t_{1}}{\Delta t}\right\|^{p} d t \\
& +\sum_{n=2}^{N} \int_{t_{n-1}}^{t_{n}}\left\|\frac{3}{2} u^{n}-\frac{1}{2} u^{n-1}+\left(\frac{3}{2} u^{n}-2 u^{n-1}+\frac{1}{2} u^{n-2}\right) \frac{t-t_{n}}{\Delta t}\right\|^{p} d t \leq c \Delta t \sum_{n=0}^{N}\left\|u^{n}\right\|^{p} .
\end{aligned}
$$

Note that the sequence of bounds $M\left(u_{\Delta t}^{0}, f\right)$ in (3.4) is bounded because of (4.2).

The theorems of Eberlein-Šmulyan and Banach-Alaoglu (see [8, Cor. III.26, Thm. III.27]), density arguments, and the compactness result of Lions-Aubin (see [21, Thm. 5.1 on p. 58]) then allow us to extract a subsequence (still denoted by the subscript $\Delta t$ ) such that

$$
\begin{aligned}
& u_{\Delta t} \rightarrow u \text { in } L^{p}(0, T ; V), u_{\Delta t} \stackrel{*}{\rightarrow} u \text { in } L^{\infty}(0, T ; H), \\
& v_{\Delta t} \rightarrow v \text { in } L^{p}(0, T ; V), v_{\Delta t} \stackrel{*}{\rightarrow} v \text { in } L^{\infty}(0, T ; H), \\
& v_{\Delta t}^{\prime} \rightarrow v^{\prime} \text { in } L^{p^{*}}\left(0, T ; V^{*}\right), v_{\Delta t} \rightarrow v \text { in } L^{r}(0, T ; H) \forall r \in[1, \infty)
\end{aligned}
$$


with limit functions $u \in L^{p}(0, T ; V) \cap L^{\infty}(0, T ; H), v \in \mathcal{W}^{p, p^{*}}(0, T)$.

We show that the limits $u$ and $v$ coincide. Inserting the definitions of $u_{\Delta t}$ and $v_{\Delta t}$, we find

$$
u_{\Delta t}(t)-v_{\Delta t}(t)=-\left\{\begin{array}{l}
\left(u_{\Delta t}^{1}-u_{\Delta t}^{0}\right) \frac{t-t_{1 / 2}}{\Delta t} \text { if } t \in\left[0, t_{1}\right], \\
\left(\frac{3}{2} u_{\Delta t}^{n}-2 u_{\Delta t}^{n-1}+\frac{1}{2} u_{\Delta t}^{n-2}\right) \frac{t-t_{n-1 / 2}}{\Delta t} \\
-\frac{1}{4}\left(u_{\Delta t}^{n}-2 u_{\Delta t}^{n-1}+u_{\Delta t}^{n-2}\right) \text { if } t \in\left(t_{n-1}, t_{n}\right](n=2,3, \ldots, N),
\end{array}\right.
$$

where $t_{n-1 / 2}=(n-1 / 2) \Delta t(n=1,2, \ldots, N)$. With the continuous embedding $V \hookrightarrow H$ and Hölder's inequality, we thus obtain

$$
\begin{aligned}
& \left\|u_{\Delta t}-v_{\Delta t}\right\|_{L^{p^{*}}\left(0, T ; V^{*}\right)}^{p^{*}} \leq c \Delta t\left\|u_{\Delta t}^{1}-u_{\Delta t}^{0}\right\|_{*}^{p^{*}}+c \Delta t \sum_{n=2}^{N}\left\|\frac{3}{2} u_{\Delta t}^{n}-2 u_{\Delta t}^{n-1}+\frac{1}{2} u_{\Delta t}^{n-2}\right\|_{*}^{p^{*}} \\
& +c \Delta t \sum_{n=2}^{N}\left\|u_{\Delta t}^{n}-2 u_{\Delta t}^{n-1}+u_{\Delta t}^{n-2}\right\|_{*}^{p^{*}} \\
& \leq c(\Delta t)^{1+p^{*}}\left\|\frac{1}{\Delta t}\left(u_{\Delta t}^{1}-u_{\Delta t}^{0}\right)\right\|_{*}^{p^{*}} \\
& +c(\Delta t)^{1+p^{*}} \sum_{n=2}^{N}\left\|\frac{1}{\Delta t}\left(\frac{3}{2} u_{\Delta t}^{n}-2 u_{\Delta t}^{n-1}+\frac{1}{2} u_{\Delta t}^{n-2}\right)\right\|_{*}^{p^{*}} \\
& +c\left(\Delta t \sum_{n=2}^{N}\left|u_{\Delta t}^{n}-2 u_{\Delta t}^{n-1}+u_{\Delta t}^{n-2}\right|^{2}\right)^{p^{*} / 2} \text {. }
\end{aligned}
$$

The a priori estimates of Theorem 3.1 now provide the strong convergence

$$
u_{\Delta t}-v_{\Delta t} \rightarrow 0 \text { in } L^{p^{*}}\left(0, T ; V^{*}\right),
$$

which shows by density that $u=v$.

Lemma 4.3. Under the assumptions of Theorem 4.1, there is a subsequence, denoted by $k^{\prime}$, such that

$$
u_{(\Delta t)_{k^{\prime}}}^{1}-u_{(\Delta t)_{k^{\prime}}}^{0} \rightarrow 0 \text { in } H
$$

Proof. We again omit the subscript $k$. From (3.6), we already know that

$$
\left|u_{\Delta t}^{1}\right|^{2}+\left|u_{\Delta t}^{1}-u_{\Delta t}^{0}\right|^{2}+\mu \Delta t|| u_{\Delta t}^{1} \|^{p} \leq c\left(\left|u_{\Delta t}^{0}\right|^{2}+\Delta t\left\|f_{\Delta t}^{1}\right\|_{*}^{p^{*}}\right) .
$$

Since $u_{\Delta t}^{0} \rightarrow u_{0}$ in $H$, the sequence $\left\{u_{\Delta t}^{0}\right\}$ is bounded in $H$. Moreover, Hölder's inequality yields

$$
\Delta t\left\|f_{\Delta t}^{1}\right\|_{*}^{p^{*}}=\Delta t\left\|\frac{1}{\Delta t} \int_{0}^{\Delta t} f(t) d t\right\|_{*}^{p^{*}} \leq \int_{0}^{\Delta t}\|f(t)\|_{*}^{p^{*}} d t
$$


with the right-hand side converging towards zero since $f \in L^{p^{*}}\left(0, T ; V^{*}\right)$. Hence, the sequence $\left\{u_{\Delta t}^{1}-u_{\Delta t}^{0}\right\}$ is bounded in $H$, and so there is a subsequence and an element $\xi \in H$ such that

$$
u_{\Delta t}^{1}-u_{\Delta t}^{0} \rightarrow \xi \text { in } H
$$

On the other hand, the second estimate in (3.4) shows that

$$
\left\|u_{\Delta t}^{1}-u_{\Delta t}^{0}\right\|_{*} \leq c(\Delta t)^{1 / p}
$$

and, therefore,

$$
u_{\Delta t}^{1}-u_{\Delta t}^{0} \rightarrow 0 \text { in } V^{*} .
$$

By density, we thus have $\xi=0$.

We now prove that not only weak but strong convergence of $u_{\Delta t}^{1}-u_{\Delta t}^{0}$ towards zero in $H$ takes place. With (2.1) and the monotonicity of $A$, we find for any $w \in V$

$$
\begin{aligned}
\left(u_{\Delta t}^{1}-u_{\Delta t}^{0}, u_{\Delta t}^{0}\right) & =\left(u_{\Delta t}^{1}-u_{\Delta t}^{0}, u_{\Delta t}^{1}\right)-\left|u_{\Delta t}^{1}-u_{\Delta t}^{0}\right|^{2} \\
& \leq\left(u_{\Delta t}^{1}-u_{\Delta t}^{0}, u_{\Delta t}^{1}\right) \\
& =\Delta t\left\langle f_{\Delta t}^{1}, u_{\Delta t}^{1}\right\rangle-\Delta t\left\langle A u_{\Delta t}^{1}, u_{\Delta t}^{1}\right\rangle-\Delta t\left\langle B u_{\Delta t}^{1}, u_{\Delta t}^{1}\right\rangle \\
& \leq \Delta t\left\langle f_{\Delta t}^{1}, u_{\Delta t}^{1}\right\rangle-\Delta t\left\langle A u_{\Delta t}^{1}, u_{\Delta t}^{1}\right\rangle+\Delta t\left\langle A u_{\Delta t}^{1}-A w, u_{\Delta t}^{1}-w\right\rangle \\
& =\Delta t\left\langle f_{\Delta t}^{1}, u_{\Delta t}^{1}\right\rangle-\Delta t\left\langle A u_{\Delta t}^{1}, w\right\rangle-\Delta t\left\langle A w, u_{\Delta t}^{1}-w\right\rangle .
\end{aligned}
$$

Because of the weak convergence of $u_{\Delta t}^{1}-u_{\Delta t}^{0}$ towards zero and the strong convergence of $u_{\Delta t}^{0}$ towards $u_{0}$, both in $H$, we obtain

$$
\left(u_{\Delta t}^{1}-u_{\Delta t}^{0}, u_{\Delta t}^{0}\right) \rightarrow 0
$$

We also observe that

$$
\Delta t\left|\left\langle f_{\Delta t}^{1}, u_{\Delta t}^{1}\right\rangle\right| \leq\left(\Delta t\left\|f_{\Delta t}^{1}\right\|_{*}^{p^{*}}\right)^{1 / p^{*}}\left(\Delta t\left\|u_{\Delta t}^{1}\right\|^{p}\right)^{1 / p} .
$$

Since the first factor of the right-hand side converges towards zero and the second one is bounded (see (3.4)), we find

$$
\Delta t\left\langle f_{\Delta t}^{1}, u_{\Delta t}^{1}\right\rangle \rightarrow 0
$$

Furthermore, we infer from the growth condition for $A$ that

$$
\begin{gathered}
\Delta t\left|\left\langle A u_{\Delta t}^{1}, w\right\rangle\right| \leq \Delta t\left\|A u_{\Delta t}^{1}\right\|_{*}\|w\| \\
\leq c \Delta t\left(1+\left\|u_{\Delta t}^{1}\right\|^{p-1}\right)\|w\| \leq c \Delta t\|w\|+c(\Delta t)^{1 / p}\left(\Delta t\left\|u_{\Delta t}^{1}\right\|^{p}\right)^{1 / p^{*}},
\end{gathered}
$$

and thus

$$
\Delta t\left\langle A u_{\Delta t}^{1}, w\right\rangle \rightarrow 0
$$

We also find

$$
\Delta t\left|\left\langle A w, u_{\Delta t}^{1}-w\right\rangle\right| \leq \Delta t\|A w\|_{*}\left\|u_{\Delta t}^{1}-w\right\|=(\Delta t)^{1 / p^{*}}\|A w\|_{*}\left(\Delta t\left\|u_{\Delta t}^{1}-w\right\|^{p}\right)^{1 / p}
$$


and thus, again with (3.4),

$$
\Delta t\left\langle A w, u_{\Delta t}^{1}-w\right\rangle \rightarrow 0 .
$$

This, finally, shows, because of (4.4), that

$$
\left(u_{\Delta t}^{1}-u_{\Delta t}^{0}, u_{\Delta t}^{1}\right) \rightarrow 0
$$

and yields, because of (4.5),

$$
\left|u_{\Delta t}^{1}-u_{\Delta t}^{0}\right|^{2}=\left(u_{\Delta t}^{1}-u_{\Delta t}^{0}, u_{\Delta t}^{1}\right)-\left(u_{\Delta t}^{1}-u_{\Delta t}^{0}, u_{\Delta t}^{0}\right) \rightarrow 0 .
$$

The proof for the semi-implicit variant is analogous and relies upon a modification in (4.4) employing $\left\langle B\left(u_{\Delta t}^{0}, u_{\Delta t}^{1}\right), u_{\Delta t}^{1}\right\rangle=0$ instead of $\left\langle B u_{\Delta t}^{1}, u_{\Delta t}^{1}\right\rangle=0$.

We are now ready to prove the main result.

Proof. [Proof of Theorem 4.1] For readability, we omit the subscripts $k$ and $k^{\prime}$.

First, we consider the numerical scheme (3.2), which can be rewritten as the differential equation

$$
v_{\Delta t}^{\prime}+A u_{\Delta t}+B u_{\Delta t}=f_{\Delta t},
$$

where $f_{\Delta t}$ denotes the piecewise constant interpolation of $\left\{f_{\Delta t}^{n}\right\}$.

By standard arguments, we find

$$
f_{\Delta t} \rightarrow f \text { in } L^{p^{*}}\left(0, T ; V^{*}\right) .
$$

The growth condition for $A$ (see Lemma2.3) together with the boundedness of $\left\{u_{\Delta t}\right\}$ in $L^{p}(0, T ; V)$ (this was a consequence of the a priori estimates (3.4) in Theorem 3.1, see also Lemma 4.2) provides the boundedness of $\left\{A u_{\Delta t}\right\}$ in $L^{p^{*}}\left(0, T ; V^{*}\right)$. Thus, there is an element $a \in L^{p^{*}}\left(0, T ; V^{*}\right)$ and a subsequence (still denoted by the subscript $\Delta t$ ) such that

$$
A u_{\Delta t} \rightarrow a \text { in } L^{p^{*}}\left(0, T ; V^{*}\right) .
$$

From Lemma 4.2, we already know that (again for a subsequence)

$$
v_{\Delta t}^{\prime} \rightarrow u^{\prime} \text { in } L^{p^{*}}\left(0, T ; V^{*}\right)
$$

for some $u \in \mathcal{W}^{p, p^{*}}(0, T)$. Indeed, we will show that $u$ solves the original initial-value problem (3.1). For the convection term with $B$, we observe that

$$
B u_{\Delta t} \rightarrow B u \text { in } L^{p^{*}}\left(0, T ; V^{*}\right) .
$$

This can be seen as follows. Because of the growth condition (2.6) from Lemma 2.4, $\left\{B u_{\Delta t}-B u\right\}$ is bounded in $L^{p^{*}}\left(0, T ; V^{*}\right)$. The subsequence can thus be chosen in such a way that

$$
B u_{\Delta t}-B u \rightarrow b \text { in } L^{p^{*}}\left(0, T ; V^{*}\right)
$$

for some $b \in L^{p^{*}}\left(0, T ; V^{*}\right)$. On the other hand, estimate (2.7) from Lemma 2.4 together with the strong convergence $u_{\Delta t} \rightarrow u$ in any $L^{r}(0, T ; H)$ for $r \in[1, \infty)$ (see Lemma 4.2) provides the strong convergence

$$
B u_{\Delta t} \rightarrow B u \text { in } L^{q}\left(0, T ; V^{*}\right)
$$


for all sufficiently small $q>1$. (Indeed, we can choose $q=p^{*}$ if $p>\max (2, d)$ giving strong convergence in (4.10).) Since $L^{q^{*}}(0, T ; V)$ is dense in $L^{p}(0, T ; V)$, we conclude with $b=0$.

Finally, we obtain from (4.6) in the limit the equation

$$
u^{\prime}+a+B u=f \text { in } L^{p^{*}}\left(0, T ; V^{*}\right),
$$

and it remains to prove that $a=A u$ and $u(0)=u_{0}$.

In proving $u(0)=u_{0}$, we observe the following. Since $\left\{v_{\Delta t}\right\}$ is bounded in $\mathcal{W}^{p, p^{*}}(0, T) \hookrightarrow \mathcal{C}([0, T] ; H)$, the sequence $\left\{v_{\Delta t}(t)\right\}$ is bounded in $H$ for any $t \in[0, T]$. With (4.2) and Lemma 4.3, we have for a suitable subsequence

$$
v_{\Delta t}(0)=\frac{1}{2}\left(u_{\Delta t}^{1}+u_{\Delta t}^{0}\right) \rightarrow u_{0} \text { in } H
$$

as well as

$$
v_{\Delta t}(T)=\frac{3}{2} u_{\Delta t}^{N}-\frac{1}{2} u_{\Delta t}^{N-1} \rightarrow \theta \text { in } H
$$

for some $\theta \in H$. Since $u, v_{\Delta t} \in \mathcal{W}^{p, p^{*}}(0, T)$, we can employ integration by parts and, with (4.11) and (4.6), we obtain for all $v \in V$ and $\phi \in \mathcal{C}^{1}([0, T])$

$$
\begin{gathered}
(u(T), v) \phi(T)-(u(0), v) \phi(0)=\left\langle u^{\prime}, v \phi\right\rangle+\left\langle v \phi^{\prime}, u\right\rangle=\langle f-a-B u, v \phi\rangle+\left\langle v \phi^{\prime}, u\right\rangle \\
=\left\langle f-f_{\Delta t}+v_{\Delta t}^{\prime}+A u_{\Delta t}+B u_{\Delta t}-a-B u, v \phi\right\rangle+\left\langle v \phi^{\prime}, u\right\rangle \\
=\left\langle f-f_{\Delta t}+A u_{\Delta t}-a+B u_{\Delta t}-B u, v \phi\right\rangle+\left\langle v \phi^{\prime}, u-v_{\Delta t}\right\rangle \\
+\left(v_{\Delta t}(T), v\right) \phi(T)-\left(v_{\Delta t}(0), v\right) \phi(0) .
\end{gathered}
$$

Remember here that the dual pairing $\langle\cdot, \cdot\rangle$ is given by

$$
\langle g, w\rangle=\int_{0}^{T}\langle g(t), w(t)\rangle_{V^{*} \times V} d t, \quad w \in L^{p}(0, T ; V), g \in L^{p^{*}}\left(0, T ; V^{*}\right) .
$$

Taking the limit on the right-hand side of (4.12) and invoking (4.7), (4.8), (4.10) as well as $v_{\Delta t} \rightarrow u$ in $L^{p}(0, T ; V)$ (see Lemma 4.2), we come up with

$$
(u(T), v) \phi(T)-(u(0), v) \phi(0)=(\theta, v) \phi(T)-\left(u_{0}, v\right) \phi(0) .
$$

Choosing $\phi(T)=0$ and $\phi(0)=0$, respectively, we find that $u(0)=u_{0}$ and $u(T)=\theta$ in $H$ since $V \ni v$ is dense in $H$, i.e.

$$
v_{\Delta t}(0) \rightarrow u(0)=u_{0} \text { in } H, \quad v_{\Delta t}(T) \rightarrow u(T) \text { in } H .
$$

For proving $a=A u$, we adapt Minty's monotonicity trick. Usually, this would require strong convergence in (4.10). However, because of $\langle B v, v\rangle=0$ for all $v \in$ $L^{p}(0, T ; V) \cap L^{\infty}(0, T ; H)$ (see $(2.1)$ ), the weak convergence suffices.

Testing (4.6) by $u_{\Delta t}$ and employing the monotonicity of $A$, we obtain for all $w \in L^{p}(0, T ; V)$

$$
\begin{gathered}
\left\langle v_{\Delta t}^{\prime}, u_{\Delta t}-v_{\Delta t}\right\rangle+\left\langle v_{\Delta t}^{\prime}, v_{\Delta t}\right\rangle+\left\langle A u_{\Delta t}, w\right\rangle+\left\langle A w, u_{\Delta t}-w\right\rangle= \\
\left\langle v_{\Delta t}^{\prime}, u_{\Delta t}\right\rangle+\left\langle A u_{\Delta t}, u_{\Delta t}\right\rangle-\left\langle A u_{\Delta t}-A w, u_{\Delta t}-w\right\rangle+\left\langle B u_{\Delta t}, u_{\Delta t}\right\rangle \leq\left\langle f_{\Delta t}, u_{\Delta t}\right\rangle .
\end{gathered}
$$

Because of (4.7), (4.8), and $u_{\Delta t} \rightarrow u$ in $L^{p}(0, T ; V)$ (see Lemma 4.2), we already have

$$
\left\langle A u_{\Delta t}, w\right\rangle \rightarrow\langle a, w\rangle, \quad\left\langle A w, u_{\Delta t}-w\right\rangle \rightarrow\langle A w, u-w\rangle, \quad\left\langle f_{\Delta t}, u_{\Delta t}\right\rangle \rightarrow\langle f, u\rangle .
$$


Moreover, with

$$
\begin{aligned}
\left(\frac{3}{2} a-2 b+\frac{1}{2} c\right)(a-2 b+c) & =\frac{3}{2}(a-b)^{2}-2(a-b)(b-c)+\frac{1}{2}(b-c)^{2} \\
& \geq \frac{1}{2}(a-b)^{2}-\frac{1}{2}(b-c)^{2}, \quad a, b, c \in \mathbb{R},
\end{aligned}
$$

and (4.3), we find

$$
\begin{aligned}
\left\langle v_{\Delta t}^{\prime}, u_{\Delta t}-v_{\Delta t}\right\rangle=\sum_{n=1}^{N} \int_{t_{n-1}}^{t_{n}}\left\langle v_{\Delta t}^{\prime}(t), u_{\Delta t}(t)-v_{\Delta t}(t)\right\rangle d t \\
=-\frac{1}{(\Delta t)^{2}} \int_{0}^{t_{1}}\left(t-t_{1 / 2}\right) d t\left|u_{\Delta t}^{1}-u_{\Delta t}^{0}\right|^{2} \\
\quad-\frac{1}{(\Delta t)^{2}} \sum_{n=2}^{N} \int_{t_{n-1}}^{t_{n}}\left(t-t_{n-1 / 2}\right) d t\left|\frac{3}{2} u_{\Delta t}^{n}-2 u_{\Delta t}^{n-1}+\frac{1}{2} u_{\Delta t}^{n-2}\right|^{2} \\
\quad+\frac{1}{4} \sum_{n=2}^{N}\left(\frac{3}{2} u_{\Delta t}^{n}-2 u_{\Delta t}^{n-1}+\frac{1}{2} u_{\Delta t}^{n-2}, u_{\Delta t}^{n}-2 u_{\Delta t}^{n-1}+u_{\Delta t}^{n-2}\right) \\
=\frac{1}{4} \sum_{n=2}^{N}\left(\frac{3}{2} u_{\Delta t}^{n}-2 u_{\Delta t}^{n-1}+\frac{1}{2} u_{\Delta t}^{n-2}, u_{\Delta t}^{n}-2 u_{\Delta t}^{n-1}+u_{\Delta t}^{n-2}\right) \\
\geq \frac{1}{8} \sum_{n=2}^{N}\left(\left|u_{\Delta t}^{n}-u_{\Delta t}^{n-1}\right|^{2}-\left|u_{\Delta t}^{n-1}-u_{\Delta t}^{n-2}\right|^{2}\right) \geq-\frac{1}{8}\left|u_{\Delta t}^{1}-u_{\Delta t}^{0}\right|^{2}
\end{aligned}
$$

with the right-hand side converging towards zero as was shown in Lemma 4.3.

With integration by parts (note that $v_{\Delta t} \in \mathcal{W}^{p, p^{*}}(0, T)$ ), we obtain

$$
\left\langle v_{\Delta t}^{\prime}, v_{\Delta t}\right\rangle=\frac{1}{2}\left|v_{\Delta t}(T)\right|^{2}-\frac{1}{2}\left|v_{\Delta t}(0)\right|^{2},
$$

and with (4.13), we come up with

$$
\left\langle u^{\prime}, u\right\rangle=\frac{1}{2}|u(T)|^{2}-\frac{1}{2}|u(0)|^{2} \leq \liminf \left(\frac{1}{2}\left|v_{\Delta t}(T)\right|^{2}-\frac{1}{2}\left|v_{\Delta t}(0)\right|^{2}\right)=\liminf \left\langle v_{\Delta t}^{\prime}, v_{\Delta t}\right\rangle .
$$

Altogether, we find from (4.14) with (4.11) (remember again $\langle B u, u\rangle=0$ )

$$
\left\langle u^{\prime}, u\right\rangle+\langle a, w\rangle+\langle A w, u-w\rangle \leq\langle f, u\rangle=\left\langle u^{\prime}, u\right\rangle+\langle a, u\rangle
$$

and thus

$$
\langle A w, u-w\rangle \leq\langle a, u-w\rangle .
$$

With $w=u \mp s v$ for arbitrary $v \in L^{p}(0, T ; V), s \in[-1,1]$, and the hemicontinuity of $A: L^{p}(0, T ; V) \rightarrow L^{p^{*}}\left(0, T ; V^{*}\right)$ (see Lemma 2.3), we get $a=A u$ from $s \rightarrow 0$, and the assertion is proved.

We now give the modifications of the proof necessary for the semi-implicit variant of (3.2) with (3.3). Without any further change, we have to replace $B u_{\Delta t}$ in (4.6), (4.12) as well as (4.14) by

$$
B\left(E u_{\Delta t}, u_{\Delta t}\right) \text { with } E u_{\Delta t}(t):= \begin{cases}u_{\Delta t}^{0} & \text { if } t \in\left[0, t_{1}\right], \\ 2 u_{\Delta t}^{n-1}-u_{\Delta t}^{n-2} & \text { if } t \in\left(t_{n-1}, t_{n}\right](n=2,3, \ldots, N) .\end{cases}
$$


The limit Equ. (4.11) remains true since, for a subsequence,

$$
B\left(E u_{\Delta t}, u_{\Delta t}\right)-B u_{\Delta t} \rightarrow 0 \text { in } L^{p^{*}}\left(0, T ; V^{*}\right) .
$$

This can be seen as follows. We have

$$
B\left(E u_{\Delta t}, u_{\Delta t}\right)-B u_{\Delta t}=B\left(w_{\Delta t}, u_{\Delta t}\right)
$$

with

$$
w_{\Delta t}(t):=E u_{\Delta t}-u_{\Delta t}=- \begin{cases}u_{\Delta t}^{1}-u_{\Delta t}^{0} & \text { if } t \in\left[0, t_{1}\right], \\ u_{\Delta t}^{n}-2 u_{\Delta t}^{n-1}+u_{\Delta t}^{n-2} & \text { if } t \in\left(t_{n-1}, t_{n}\right](n=2,3, \ldots, N) .\end{cases}
$$

Because of the first a priori estimate in (3.4) of Theorem 3.1, we easily find

$$
w_{\Delta t} \rightarrow 0 \text { in } L^{2}(0, T ; H)
$$

and, hence, in any $L^{r}(0, T ; H)$ with $r \in[1, \infty)$ since $\left\{w_{\Delta t}\right\}$ is bounded in $L^{\infty}(0, T ; H)$. This yields, with an estimate analogous to $(2.7)$,

$$
B\left(w_{\Delta t}, u_{\Delta t}\right) \rightarrow 0 \text { in } L^{q}\left(0, T ; V^{*}\right)
$$

for sufficiently small $q>1$. By growth estimates, we also know that $\left\{B\left(w_{\Delta t}, u_{\Delta t}\right)\right\}$ is bounded in $L^{p^{*}}\left(0, T ; V^{*}\right)$ such that a subsequence is weakly convergent in $L^{p^{*}}\left(0, T ; V^{*}\right)$. By density arguments, the limit can only be zero.

\section{REFERENCES}

[1] G. Akrivis and M. Crouzeix, Linearly implicit methods for nonlinear parabolic equations, Math. Comp., 73(246), 613-635, 2004.

[2] G. Akrivis, M. Crouzeix and C. Makridakis, Implicit-explicit multistep finite element methods for nonlinear parabolic problems, Math. Comp., 67(222), 457-477, 1998.

[3] G. Akrivis, M. Crouzeix and C. Makridakis, Implicit-explicit multistep methods for quasilinear parabolic equations, Numer. Math., 82(4), 521-541, 1999.

[4] G. Astarita and G. Marrucci, Principles of Non-Newtonian Fluid Mechanics, McGraw-Hill, New York, 1974.

[5] J.W. Barrett and E. Süli, Existence of global weak solutions to some regularized kinetic models for dilute polymers, Multiscale Model. Simul., 6(2), 506-546, 2007.

[6] J.W. Barrett and E. Süli, Existence of global weak solutions to dumbbell models for dilute polymers with microscopic cut-off, M3AS, 18(6), 935-971, 2008.

[7] R.B. Bird, R.C. Armstrong and O. Hassager, Dynamics of Polymer Liquids, Wiley, New York, 1987.

[8] H. Brézis, Analyse Fonctionnelle: Théorie et Applications, Dunod, Paris, 1999.

[9] L. Diening, A. Prohl and M. Růžička, Semi-implicit Euler scheme for generalized Newtonian fluids, SIAM J. Numer. Anal., 44(3), 1172-1190, 2006.

[10] L. Diening, M. Růžička and J. Wolf, Existence of weak solutions for unsteady motions of generalized Newtonian fluids, Universität Freiburg, Mathematisches Institut, Preprint 0802, 2008.

[11] E. Emmrich, Stability and convergence of the two-step BDF for the incompressible NavierStokes problem, Int. J. Nonlinear Sci. Numer. Simul., 5(3), 199-210, 2004.

[12] E. Emmrich, Error of the two-step BDF for the incompressible Navier-Stokes problem, M2AN, 38(5), 757-764, 2004.

[13] E. Emmrich, Two-step BDF time discretisation of nonlinear evolution problems governed by monotone operators with strongly continuous perturbations, to appear in Comput. Meth. Appl. Math., 2008.

[14] E. Fernández-Cara, F. Guillén and R.R. Ortega, Mathematical modeling and analysis of viscoelastic fluids of the Oldroyd kind, P.G. Ciarlet, J.L. Lions (eds.), Handbook of numerical analysis, vol. VIII, Numerical methods for fluids (Part 2), Elsevier, Amsterdam, 2002. 
[15] B. García-Archilla, Some practical experience with the time integration of dissipative equations, J. Comput. Phys., 122(1), 25-29, 1995.

[16] B. García-Archilla and J. de Frutos, Time integration of the nonlinear Galerkin method, IMA J. Numer. Anal., 15(2), 221-244, 1995.

[17] E. Hairer and G. Wanner, Solving Ordinary Differential Equations, II: Stiff and DifferentialAlgebraic Problems, Springer, Berlin, 1991.

[18] V. John, G. Matthies and J. Rang, A comparison of time-discretization/linearization approaches for the incompressible Navier-Stokes equations, Comput. Methods Appl. Mech. Engrg., 196, 5995-6010, 2006.

[19] B. Jourdain, C. Le Bris, T. Lelièvre and F. Otto, Long-time asymptotics of a multiscale model for polymeric fluid flows, Arch. Rational Mech. Anal., 181, 97-148, 2006.

[20] R. Keunings, Micro-macro methods for the multiscale simulation of viscoelastic flow using molecular models of kinetic theory, Rheol. Rev., 67-98, 2004.

[21] J.L. Lions, Quelques Méthodes de Résolution des Problèmes aux Limites non Linéaires, Dunod, Gauthier-Villars, Paris, 1969.

[22] P. Le Tallec, Numerical Analysis of Viscoelastic Problems, Masson, Paris, 1990.

[23] J. Málek, J. Nečas, M. Rokyta and M. Růžička, Weak and measure-valued solutions to evolutionary PDEs, Chapman \& Hall, London, 1996.

[24] M. Marion and R. Temam, Navier-Stokes equations: Theory and Approximation, P.G. Ciarlet, J.-L. Lions (eds.), Handbook of numerical analysis, vol. VI, Numerical methods for fluids (Part 1), Elsevier, Amsterdam, 1998.

[25] A. Mellet and A. Vasseur, Global weak solutions for a Vlasov-Fokker-Planck/Navier-Stokes system of equations, M3AS, 17(7), 1039-1063, 2007.

[26] A. Prohl, M. Růžička, On fully implicit space-time discretization for motions of incompressible fluids with shear-dependent viscosities: the case $p \leq 2$, SIAM J. Numer. Anal., 39(1), 214249, 2001.

[27] M. Reiner, Rheologie in Elementarer Darstellung, Carl Hanser, München, 1968.

[28] M. Renardy, Mathematical Analysis of Viscoelastic Flows, SIAM, Philadelphia, 2000.

[29] R. Temam, Navier-Stokes equations: Theory and Numerical Analysis, North-Holland Publ. Co., Amsterdam, 1977.

[30] V. Thomée, Galerkin Finite Element Methods for Parabolic Problems, Springer, Berlin, 2006.

[31] J. Wolf, Existence of weak solutions to the equations of non-stationary motion of nonNewtonian fluids with shear rate dependent viscosity, J. Math. Fluid. Mech., 9, 104-138, 2007.

[32] E. Zeidler, Nonlinear Functional Analysis and Its Applications, II/B: Nonlinear Monotone Operators, Springer, New York, 1990. 